\title{
Collective enhancement of precision in networks of coupled oscillators
}

\author{
Daniel J. Needleman $^{\mathrm{a}, 1}$, Paul H.E. Tiesinga ${ }^{\mathrm{b}, *}$, Terrence J. Sejnowski $^{\mathrm{a}, \mathrm{b}, \mathrm{c}}$ \\ ${ }^{a}$ Howard Hughes Medical Institute, Salk Institute, La Jolla, CA 92037, USA \\ b Sloan-Swartz Center for Theoretical Neurobiology, Salk Institute, La Jolla, CA 92037, USA \\ ${ }^{c}$ Department of Biology, University of California, San Diego, La Jolla, CA 92093, USA \\ Received 10 September 2000; received in revised form 12 March 2001; accepted 28 March 2001 \\ Communicated by J.P. Keener
}

\begin{abstract}
We analyze conditions under which the precision of coupled oscillators can be improved by synchronization. The improvement in precision, defined as the inverse of the coefficient of variation of the periods, depends on how noise is added to the system. If the magnitude of noise experienced by an oscillator only depends on the state of that oscillator, then the precision of the group rhythm can be improved to $\sqrt{N}$ times the precision of an individual uncoupled oscillator, irrespective of the form of the coupling function. However, if the magnitude of the noise also depends on the state of other oscillators, as might be the case for noise caused by synaptic input to neurons, then synchronization may lead to an additional improvement. This collective enhancement of precision is demonstrated both with a simple phase model and a network of integrate-and-fire neurons. (C) 2001 Elsevier Science B.V. All rights reserved.
\end{abstract}

PACS: 87.10.+e

Keywords: Synchronization; Rhythm; Noise; Neuron

\section{Introduction}

Coupled oscillators are found throughout science and include arrays of Josephson junctions, circadian rhythms, flashing fireflies, electronic circuits, and neuronal networks [1-12]. In these systems the coupling between different oscillators may force them to synchronize to a constant frequency. All real systems contain noise, so real oscillators possess some degree of jitter and thus have limited precision. Intuitively, it seems possible that synchronization might discipline the oscillators to become more precise $[1,13,14]$. Researchers have proposed that this phenomenon, called collective enhancement of precision [1], might explain the remarkable precision of some biological signals such as circadian rhythms $[1,2,15,16]$ and the electric organ discharge of weakly electric fishes [16-19].

It has been claimed that collective enhancement of precision may be one of the purposes of synchronized neural activity [20], and evidence that the reliability of neurons improves with increased synaptic efficacy [21] provides

\footnotetext{
* Corresponding author. Tel.: +1-858-453-4100, ext. 1039; fax: +1-858-455-7933.

E-mail address: tiesinga@salk.edu (P.H.E. Tiesinga).

${ }^{1}$ Current address: Department of Physics, University of California, Santa Barbara, CA 93117, USA.
} 
support for this hypothesis. Furthermore, this type of increase in precision can be used to construct improved electronic clocks $[22,23]$. However, to our knowledge, it has never been demonstrated that the precision of coupled oscillators can be improved beyond $\sqrt{N}$, which results from averaging the frequencies of the uncoupled oscillators. Thus it is not clear if network effects can lead to an additional increase in precision beyond that obtained by simple averaging. In this paper we show that the manner in which noise enters the system is critical in determining the limits of collective enhancement of precision.

We define the precision of a population of oscillators as the jitter in the average spike time of all the oscillators in the network during one cycle. It is helpful to make a distinction between "intrinsic" noise and "extrinsic" noise. An oscillator is said to possess intrinsic noise if the resulting fluctuations in its frequency depend only on the state of that oscillator. If the resulting fluctuations in the frequency also depend on the state of other oscillators then the noise is called extrinsic. For neurons, ion channel noise is a source of intrinsic noise. Correlated synaptic input to the neuron would be a source of extrinsic noise because the resulting fluctuation in the frequency of the neuron is a function of the relative phase between the neuron and the input.

\section{Intrinsic noise: phase model}

First we determine the effect of collective enhancement of precision in systems of coupled oscillators with intrinsic noise. A limit cycle oscillator that is weakly coupled to other limit cycle oscillators can be described by a single phase variable, with interactions between the oscillators expressed as a function of the difference of the phases. For intrinsically noisy limit cycle oscillators subjected to Gaussian white noise, an additive fluctuating noise term is included. Consider the network of oscillators pictured in Fig. 1 as an example of a system with intrinsic noise. This system consists of two layers of oscillators, the top layer, in which each oscillator keeps a constant frequency, and the bottom layer, where each oscillator is coupled unidirectionally to an oscillator in the top layer and coupled to all the other oscillators in the bottom layer. Thus, the phases obey an equation of the form,

$$
\dot{\theta}_{i}=\omega_{i}+H_{\mathrm{t}}\left(\phi_{i}-\theta_{i}\right)+\sum_{j \neq i}^{N} H\left(\theta_{j}-\theta_{i}\right)+\xi_{\mathrm{i}},
$$

where $\theta_{i}$ is the phase of the $i$ th oscillator, $\omega_{i}$ the oscillator's natural frequency, $\phi_{i}=\phi_{i}^{0} \omega_{t} t$ the phase of the oscillator in the top layer coupled to the $i$ th oscillator in the bottom layer with initial phase $\phi_{i}^{0}$ and frequency $\omega_{t}, N$ the number of oscillators, $H\left(\theta_{j}-\theta_{i}\right)$ is a coupling function that describes the interaction between the oscillators in the bottom layer, $H_{\mathrm{t}}\left(\phi_{i}-\theta_{i}\right)$ the coupling function connecting oscillators in the bottom layer to the top layer, $\xi_{\mathrm{i}}$ is an intrinsic Gaussian white noise term with standard deviation $Q_{\text {in }}$, and the sum is over all other oscillators except the $i$ th one.

Complete derivations of phase equations are available, see [11,24,25], here we only briefly sketch how Eq. (1) may be derived. A phase variable for each oscillator may be defined such that, for the uncoupled oscillator in the absence of noise, the asymptotic behavior of the oscillator is only a function of its current phase. Thus, the original multi-dimensional system that describes the behavior of one oscillator is replaced by a one-dimensional

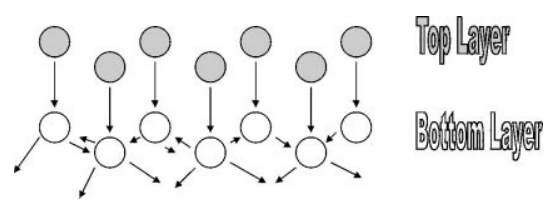

Fig. 1. A diagram showing the connections in the network of oscillators discussed in the text. The oscillators in the top layer maintain a constant frequency and connect one-to-one with oscillators in the bottom layer. The oscillators in the bottom layer have all-to-all coupling. The noise is either added as Gaussian noise to the bottom layer, or inserted as jitter in the input from oscillators in the top layer to oscillators in the bottom layer. In the latter case the oscillators in the bottom layer are extrinsically noisy because the magnitude of jitter in their frequency depends on the relative phase of the input from the top layer. 
parameterization along the oscillators' limit cycle. Then the coupling functions and the magnitude of the noise $Q_{\text {in }}$ are derived perturbatively by assuming the oscillator stays on its unperturbed limit cycle and then averaging the effect of coupling and noise over one period [11,24,26]. Eq. (1) is only applicable for an oscillator with intrinsic noise because the magnitude of $Q_{\text {in }}$ is obtained by integrating the effect of the noise over one period of the oscillators' unperturbed trajectory, and that procedure is only valid if the integrand is solely a function of the state of the oscillator.

Phase models have been successfully derived for a number of systems including arrays of Josephson junctions [6] and biophysically detailed Hodgkin-Huxley neurons [4,5]. Phase equations of the same form can also be derived for integrate-and-fire neurons [27,28]. Experimental tests of phase models, in the lamprey locomotor central pattern generator [8] and the mollusk Limax maximus's olfactory lobe [9], for example, have shown that these models can have predictive and explanatory power.

We first consider the case $H_{\mathrm{t}}\left(\phi_{i}-\theta_{i}\right)=0$. To determine the behavior of the group rhythm, $\Omega=(1 / N) \sum_{i} \dot{\theta}_{i}$, of the intrinsically noisy oscillators, sum Eq. (1) over all $i$, and normalize by dividing by $N$, to obtain

$$
\Omega=\bar{\omega}+\frac{1}{N} \sum_{i}^{N} \sum_{j \neq i}^{N} H\left(\theta_{j}-\theta_{i}\right)+\Xi
$$

where $\bar{\omega}$ is the mean of the individual $\omega_{i}$ 's and $\Xi$ is a Gaussian white noise term, with a standard deviation $\sigma=Q_{\text {in }} / \sqrt{N}$, that was obtained by summing the $\xi_{\text {i }}$ 's from Eq. (1). The precision of a population of neurons is defined as the inverse of the jitter in the average spike time of all the neurons in the network during one cycle. The spike-time jitter in a single neuron is proportional to the noise current, or equivalently, to the stochastic fluctuations in the time derivative of the membrane potential. The corresponding quantity in phase models is $\sigma$, which is studied in this section and the next.

If $H\left(\theta_{j}-\theta_{i}\right)$ is an odd function, then all of the coupling terms cancel and the $\sigma$ of the group frequency is reduced to $1 / \sqrt{N}$ times the $\sigma$ of an individual oscillator [29]. If $H\left(\theta_{j}-\theta_{i}\right)$ is not odd and if in the absence of noise the sum of the coupling terms can be represented as a constant (this will be the case for any phase-locked solution), then in the presence of small noise the sum of the coupling terms will fluctuate around a constant value, assuming the equilibrium is stable. In this case the only way for $\sigma$ to be less than $Q_{\text {in }} / \sqrt{N}$ is if the fluctuations in the coupling term are correlated with the fluctuations in $\Xi$. However, this is not possible, because at any given time, $t$, the value of the coupling term is only a function of the current positions of the oscillators, while the value of $\Xi$ is uncorrelated from one instant to the next. Therefore, if $H\left(\theta_{j}-\theta_{i}\right)$ is not odd, the coupling term might lead to additional fluctuations in $\Omega$, but the $\sigma$ of the group rhythm cannot be less than $Q_{\text {in }} / \sqrt{N}$. Thus, for intrinsically noisy oscillators, the precision of the group frequency cannot be improved beyond $\sqrt{N}$. The above analysis and conclusion is also valid for $H_{\mathrm{t}}\left(\phi_{i}-\theta_{i}\right) \neq 0$. Briefly, this again leads to Eq. (2) with an additional term $H_{\mathrm{t}}\left(\phi_{i}-\theta_{i}\right)$. This extra term is also uncorrelated with $\Xi$.

A more general and formal argument is as follows. Consider a phase model where the dynamical equations can be written in terms of a Hamiltonian $\mathcal{H}$, such that $\dot{\theta}_{i}=-\partial \mathcal{H} / \partial \theta_{i}$. A phase-locked solution corresponds to a fixed point of these equations with $\theta_{i}(t)=\omega t+\beta_{i}$, here $\beta_{i}$ is constant. Without loss of generality we may transform to a rotating coordinate frame and thus set $\omega=0$. The stability of the fixed point solution is determined by the matrix of the second derivatives of $\mathcal{H}$ given by $\mathcal{H}_{i j} \equiv \partial^{2} \mathcal{H} / \partial \theta_{i} \partial \theta_{j}$. A fixed point is stable when all the eigenvalues $\lambda_{i}$ are positive. The stochastic dynamics of the small noise-induced deviations, $\delta \theta_{i}$, from the fixed point is given by

$$
\delta \dot{\theta}_{i}=-\sum_{j} \mathcal{H}_{i j} \delta \theta_{j}+\xi_{i}
$$

Since $\mathcal{H}_{i j}$ is symmetric, the eigenvectors form an orthogonal matrix $\mathcal{M}_{i j}$, that is, $\mathcal{M}_{i j}^{-1}=\mathcal{M}_{j i}$, and the above equations in eigencoordinates $\eta_{i}$ become,

$$
\delta \dot{\eta}_{i}=-\lambda_{i} \delta \eta_{i}+\sum_{j} \mathcal{M}_{i j} \xi_{j}
$$


The solution to stochastic dynamics of a single phase variable, $\dot{\theta}=-\gamma \theta+\xi$, driven by a noise source $\xi$ with variance $Q_{\text {in }}^{2}$ is

$$
\theta(t)=\mathrm{e}^{-\gamma t} \theta(0)+\int_{0}^{t} \mathrm{~d} s \mathrm{e}^{-\gamma(t-s)} \xi(s) .
$$

We take $\theta(0)=0$ and assume $\gamma t \gg 1$,

$$
\left\langle\theta(t)^{2}\right\rangle=\int_{0}^{t} \mathrm{~d} s \int_{0}^{t} \mathrm{~d} s^{\prime} \mathrm{e}^{-\gamma\left(2 t-s-s^{\prime}\right)}\left\langle\xi(s) \xi\left(s^{\prime}\right)\right\rangle=\frac{Q_{\mathrm{in}}^{2}}{2 \gamma}\left(1-\mathrm{e}^{-2 \gamma t}\right) \approx \frac{Q_{\mathrm{in}}^{2}}{2 \gamma}
$$

and

$$
\begin{aligned}
\left\langle\dot{\theta}(t)^{2}\right\rangle & =\gamma^{2}\left\langle\theta^{2}(t)\right\rangle+\left\langle\xi^{2}(t)\right\rangle-2 \gamma\langle\xi(t) \theta(t)\rangle=\frac{\gamma}{2} Q_{\mathrm{in}}^{2}+Q_{\mathrm{in}}^{2} \delta(0)-2 \gamma \int_{0}^{\infty} h(t-s) \mathrm{e}^{-\gamma(t-s)}\langle\xi(s) \xi(t)\rangle \\
& =\frac{\gamma}{2} Q_{\mathrm{in}}^{2}+Q_{\mathrm{in}}^{2} \delta(0)-2 \gamma \int_{0}^{\infty} h(t-s) \delta(t-s)=\frac{\gamma}{2} Q_{\mathrm{in}}^{2}+Q_{\mathrm{in}}^{2} \delta(0)-\gamma Q_{\mathrm{in}}^{2}=Q_{\mathrm{in}}^{2} \delta(0)-\frac{\gamma}{2} Q_{\mathrm{in}}^{2} .
\end{aligned}
$$

Here $h$ is the Heaviside function and $h(0)$ was defined as $\frac{1}{2}$. The variance of $\dot{\theta}$ contains an infinite part, $Q_{\mathrm{in}}^{2} \delta(0)$, and a finite part $-\frac{1}{2} \gamma Q_{\mathrm{in}}^{2}$. Thus the part that is measured has a variance $Q_{\mathrm{in}}^{2}$.

We now apply this result to the full network,

$$
\left\langle\sum_{i} \delta \dot{\theta}_{i}\right\rangle=0
$$

and

$$
\begin{aligned}
\left\langle\sum_{i} \delta \dot{\theta}_{i}^{2}\right\rangle & =\left\langle\sum_{i} \sum_{j} \mathcal{M}_{i j}^{-1} \delta \eta_{j} \sum_{k} \mathcal{M}_{i k}^{-1} \delta \eta_{k}\right\rangle=Q_{\mathrm{in}}^{2}\left\langle\sum_{i j k} \mathcal{M}_{i j}^{-1} \mathcal{M}_{i k}^{-1} \delta_{j k}\right\rangle \\
& =Q_{\mathrm{in}}^{2}\left\langle\sum_{i j} \mathcal{M}_{i j}^{-1} \mathcal{M}_{j i}\right\rangle=Q_{\mathrm{in}}^{2}\left\langle\sum_{i} \delta_{i i}\right\rangle=N Q_{\mathrm{in}}^{2} .
\end{aligned}
$$

Hence, the magnitude of the jitter in the group rhythm $\Omega$ is $\sigma=Q_{\text {in }} / \sqrt{N}$.

This analysis explains the $\sqrt{N}$ improvement in precision that has been observed in a variety of models of coupled oscillators such as a phenomenological model of circadian rhythms [30], models of heart-cell clusters [31], coupled relaxation-oscillators $[15,16]$, and integrate-and-fire neurons, where it was shown that the precision of individual elements is also enhanced [20]. A $\sqrt{N}$ improvement in the precision of coupled oscillators has been observed experimentally in clusters of cultured heart cells [31] and in an electronic array of coupled ring oscillators [22,23]. Rappel and Karma [20] explained the $\sqrt{N}$ improvement in precision observed in integrate-and-fire neurons by analytically finding the power spectrum of phase oscillators with linear coupling. Our results expand on this by showing that the manner in which noise enters the system is the critical factor in determining the degree of enhanced precision. Thus the increased precision of the group rhythm cannot improve beyond $\sqrt{N}$ if the noise is intrinsic, irrespective of the form of the coupling function; however, as we show, if the noise is extrinsic the increase in precision may be further improved.

In the next two sections we present analytic results and computer simulations of two models were this does occur. First we consider a simple phase model and then we show that a similar phenomena occurs in pulse-coupled integrate-and-fire neurons, where a phase description and the small noise approximation are not valid. 


\section{Extrinsic noise: phase model, $N=2$}

We again consider the network of oscillators shown in Fig. 1. In this case, however, the noise is added as jitter in the connection between the top and bottom layers. We use this setup as a simple example of a system with extrinsic noise, but we expect a similar phenomena to hold in more realistic layered systems with more complicated patterns of connectivity, such as the mollusk Limax maximus's olfactory lobe [9] or cortical layers.

We will first examine the behavior of this system using a simple phase model. In this model the phase of the oscillators in the top layer advance with a constant frequency $\omega_{\mathrm{t}}$, and the phase of an oscillator in the bottom layer is described by the following dynamics:

$$
\dot{\theta}_{i}=\omega_{\mathrm{b}}+K_{\mathrm{t}} \sin \left(\phi_{i}-\theta_{i}+\xi_{i, \mathrm{ex}}\right)+K_{\mathrm{b}} \sum_{j \neq i}^{N} \sin \left(\theta_{j}-\theta_{i}\right),
$$

where $\theta_{i}$ is the phase of the $i$ th oscillator in the bottom layer, $\phi_{i}=\phi_{i}^{0}+\omega_{\mathrm{t}} t$ is the phase of the oscillator in the top layer coupled to the $i$ th oscillator in the bottom layer, $\phi_{i}^{0}$ is the phase at $t=0, \omega_{\mathrm{b}}$ is the natural frequency of the oscillators in the bottom layer, $K_{\mathrm{t}}$ is the strength of coupling between oscillators in the top and bottom layers, $K_{\mathrm{b}}$ is the coupling between oscillators in the bottom layer. For simplicity the coupling function is chosen to be a sine. The noise term $\xi_{\mathrm{i}, \mathrm{ex}}$ is Gaussian white noise with standard deviation $Q_{\mathrm{ex}}$. The noise term is placed inside the sine function to model the effect of phasic jitter and is thus extrinsic noise, because the magnitude of the fluctuation in $\dot{\theta}_{i}$ depends on $\left|\phi_{i}-\theta_{i}\right|$. In neurons this jitter might be caused by noise in the timing of synaptic transmission or noise added during propagation of the signal along the axon of the neurons in the top layer. Thus, in the language of Ref. [32], the input from the top layer to the bottom layer has high "reliability", but limited "precision".

In the case that the oscillators in the bottom layer are uncoupled to each other, $K_{\mathrm{b}}=0$. Then, if $K_{\mathrm{t}} \geq|\Delta \omega|=$ $\left|\omega_{\mathrm{t}}-\omega_{\mathrm{b}}\right|$ (from here on we will assume that this is the case), the oscillators in the top layer entrain the oscillators in the bottom layer and, at large times, the solution for $\theta_{i}$ approaches $\theta_{i}=\phi_{i}^{0}+\omega_{\mathrm{t}} t-\arcsin \left(\Delta \omega / K_{\mathrm{t}}\right)$. Weak noise causes the oscillators to jitter around the frequency that they maintained in the absence of noise. The jitter in the uncoupled oscillators is solved to first order in $\xi_{\text {i,ex }}$ by substituting the solution for $\theta_{i}$ obtained in the absence of noise, so $\phi_{i}-\theta_{i}=\arcsin \left(\Delta \omega / K_{\mathrm{t}}\right)$, and then expanding the sine term to first order in $\xi_{i, \text { ex }}$ around this value. Therefore, each uncoupled oscillator has a frequency $\omega_{\mathrm{t}}$ and a jitter with standard deviation $Q_{\mathrm{ex}} K_{\mathrm{t}} \cos \left(\arcsin \left(\Delta \omega / K_{\mathrm{t}}\right)\right)=$ $Q_{\mathrm{ex}} \sqrt{K_{\mathrm{t}}^{2}-\Delta \omega^{2}}$.

If the oscillators are coupled, then $K_{\mathrm{b}} \neq 0$, and the solution for $\theta_{i}$ depends on $K_{\mathrm{b}}$. For two synchronized oscillators in the absence of noise, the phase of the oscillators can be solved analytically. Taking $\phi_{1}^{0}=0$ and $\phi_{2}^{0}=-\pi$, the phases of the two coupled synchronized oscillators can be solved self-consistently by setting $\frac{1}{2}\left(\dot{\theta}_{1}+\dot{\theta}_{2}\right)=\omega_{\mathrm{t}}$ and $\frac{1}{2}\left(\dot{\theta}_{1}-\dot{\theta}_{2}\right)=0$. Combining this with Eq. (8), in the absence of noise, leads to the solution for the phases of the two oscillators, $\theta_{1}=\phi_{1}^{0}+\omega_{\mathrm{t}} t+\beta_{1}$ and $\theta_{2}=\phi_{2}^{0}+\omega_{\mathrm{t}} t+\beta_{2}$, with

$$
\begin{aligned}
& \beta_{1}=\frac{\pi}{2}+\frac{\arcsin \left(4 K_{\mathrm{b}} \Delta \omega / K_{\mathrm{t}}^{2}\right)}{2}-\arcsin \left(\frac{K_{\mathrm{t}}}{2 K_{\mathrm{b}}} \cos \left(\frac{\arcsin \left(4 K_{\mathrm{b}} \Delta \omega / K_{\mathrm{t}}^{2}\right)}{2}\right)\right), \\
& \beta_{2}=-\frac{\pi}{2}+\frac{\arcsin \left(4 K_{\mathrm{b}} \Delta \omega / K_{\mathrm{t}}^{2}\right)}{2}+\arcsin \left(\frac{K_{\mathrm{t}}}{2 K_{\mathrm{b}}} \cos \left(\frac{\arcsin \left(4 K_{\mathrm{b}} \Delta \omega / K_{\mathrm{t}}^{2}\right)}{2}\right)\right) .
\end{aligned}
$$

After changing coordinates to the rotating coordinate frame, $\theta_{i} \rightarrow \hat{\theta}_{i}=\theta_{i}+\omega_{\mathrm{t}} t+\phi_{i}^{0}$, the synchronized solutions correspond to a fixed point $\hat{\theta}_{i}=\beta_{i}$. A linear stability analysis shows that this synchronized solution is stable when $\beta_{1}$ and $\beta_{2}$ are between $\pi / 2$ and $-\pi / 2$. Because we are concerned with the effects of coupling on the behavior of the oscillators in this uniform frequency regime, it is interesting to note that, for $\Delta \omega \neq 0$, if the coupling strength, $K_{\mathrm{b}}$, 
is too large, Eqs. (9) and (10), do not give real solutions for $\beta_{1}$ and $\beta_{2}$, and therefore the oscillators can no longer synchronize with constant frequency $\omega_{\mathrm{t}}$.

The magnitude of the jitter in the group rhythm is determined by substituting this solution for $\theta_{i}$ into Eq. (8), expanding the sine term to first order in $\xi_{i, \text { ex }}$ and averaging over $i$. The resulting jitter has a standard deviation, $\sigma=\frac{1}{2} K_{\mathrm{t}} Q_{\mathrm{ex}} \sqrt{\cos ^{2}\left(\beta_{1}\right)+\cos ^{2}\left(\beta_{2}\right)}$. In the limit $K_{\mathrm{t}} / 2 K_{\mathrm{b}} \rightarrow 0$, this reduces to

$$
\sigma \approx \frac{K_{\mathrm{t}} Q_{\mathrm{ex}}}{2} \sqrt{1-\cos (2 \bar{\theta}) \cos \left(\cos (\bar{\theta}) \frac{K_{\mathrm{t}}}{K_{\mathrm{b}}}\right)}
$$

with $\bar{\theta}=\frac{1}{2} \arcsin \left(4 \Delta \omega K_{\mathrm{b}} / K_{\mathrm{t}}^{2}\right)$. When, in addition, $4 K_{\mathrm{b}} \Delta \omega / K_{\mathrm{t}}^{2} \rightarrow 0$,

$$
\sigma \approx \frac{1}{K_{\mathrm{b}}}\left(\frac{K_{\mathrm{t}}^{2} Q_{\mathrm{ex}}}{2^{3 / 2}}\right)
$$

This expression becomes exact for $\Delta \omega=0$. Because the magnitude of the jitter goes like $1 / K_{\mathrm{b}}$ in a certain limit, values of $K_{\mathrm{b}}, \Delta \omega$, and $K_{\mathrm{t}}$ can be chosen so that changing the coupling $K_{\mathrm{b}}$ to the oscillators in the bottom layer from a zero to a nonzero value, will result in an arbitrarily large reduction of the standard deviation of the jitter in the group rhythm, $\sigma$. As $K_{\mathrm{b}}$ increases, and if the approximation of large $K_{\mathrm{t}}$ is valid, the oscillators phase lock with vanishing phase difference, with $\theta_{i}$ approaching $\phi_{1}-\pi / 2=\phi_{2}+\pi / 2$ and the standard deviation $\sigma$ of the group rhythm going to zero. Therefore, the standard deviation of the jitter of the group rhythm of these extrinsically noisy oscillators can be improved when coupling is introduced beyond the improvement obtained by averaging.

Note that the standard deviation, $\sigma$, of the group rhythm will go to zero irrespective of the magnitude of the noise of the uncoupled oscillators, as long as $Q_{\mathrm{ex}}$ is small enough so that higher-order terms in $\xi_{\mathrm{i} \text {,ex }}$ can be ignored. However, this improvement in precision with increasing $K_{\mathrm{b}}$ depends on the difference between $\phi_{1}$ and $\phi_{2}$, and if $\phi_{1}=\phi_{2}$ the precision of the oscillators improves when $K_{\mathrm{b}}$ becomes increasingly negative. This improvement is not due to a generalized increase in stability. In fact, a stability analysis reveals that the system becomes more and more sensitive to the effects of intrinsic noise as the coupling increases.

\section{Extrinsic noise: phase model, $N>2$}

We now extend this example of oscillators with extrinsic noise to $N>2$. We consider Eq. (8) but with the oscillators in the bottom layer divided into two groups, the group with the index $i$ odd, $1,3, \ldots, 2 n-1$, and the group with $i$ even, $2,4, \ldots, 2 n$, with $n=\frac{1}{2} N$. An "even oscillator" connects with all odd oscillators, and an "odd oscillator" connects to all even oscillators. The oscillators in the top layer proceed at a constant rate, $\phi_{i}=\omega_{\mathrm{t}} t+\phi_{i}^{0}$, with $\phi_{i}^{0}=\Delta \phi / 2$ if $i$ is odd, and equal to $-\Delta \phi / 2$ when $i$ is even, where $\Delta \phi=\pi$. The derivation proceeds in three steps. First, we substitute an Ansatz solution in the fixed point equation to obtain two equations. Second, we use our solution for $N=2$ oscillators to solve for the jitter in the group rhythm with $N>2$. Finally, we check the stability of this solution by calculating the eigenvalues of the stability matrix $\mathcal{H}_{i j}$.

The phase dynamics of the $i$ th oscillator is

$$
\dot{\theta}_{i}=\omega_{\mathrm{b}}+K_{\mathrm{t}} \sin \left(\phi_{i}-\theta_{i}+\xi_{i, \mathrm{ex}}\right)+K_{\mathrm{b}} \sum_{j *} \sin \left(\theta_{j}-\theta_{i}\right),
$$

here $\sum_{j *}$ is the sum over even oscillators if $i$ is odd, and the sum over odd oscillators if $i$ is even.

Our Ansatz is that all the even phases $\theta_{i}$ are equal to $\theta_{\mathrm{e}}$ and all the odd ones equal to $\theta_{\mathrm{o}}$, for $\xi_{i \text {,ex }}=0$, Eq. (13) then reduces to

$$
\begin{aligned}
& \dot{\theta}_{\mathrm{o}}=\omega_{\mathrm{b}}+K_{\mathrm{t}} \sin \left(\phi_{\mathrm{o}}-\theta_{\mathrm{o}}\right)+n K_{\mathrm{b}} \sin \left(\theta_{\mathrm{e}}-\theta_{\mathrm{o}}\right), \\
& \dot{\theta}_{\mathrm{e}}=\omega_{\mathrm{b}}+K_{\mathrm{t}} \sin \left(\phi_{\mathrm{e}}-\theta_{\mathrm{e}}\right)-n K_{\mathrm{b}} \sin \left(\theta_{\mathrm{e}}-\theta_{\mathrm{o}}\right)
\end{aligned}
$$



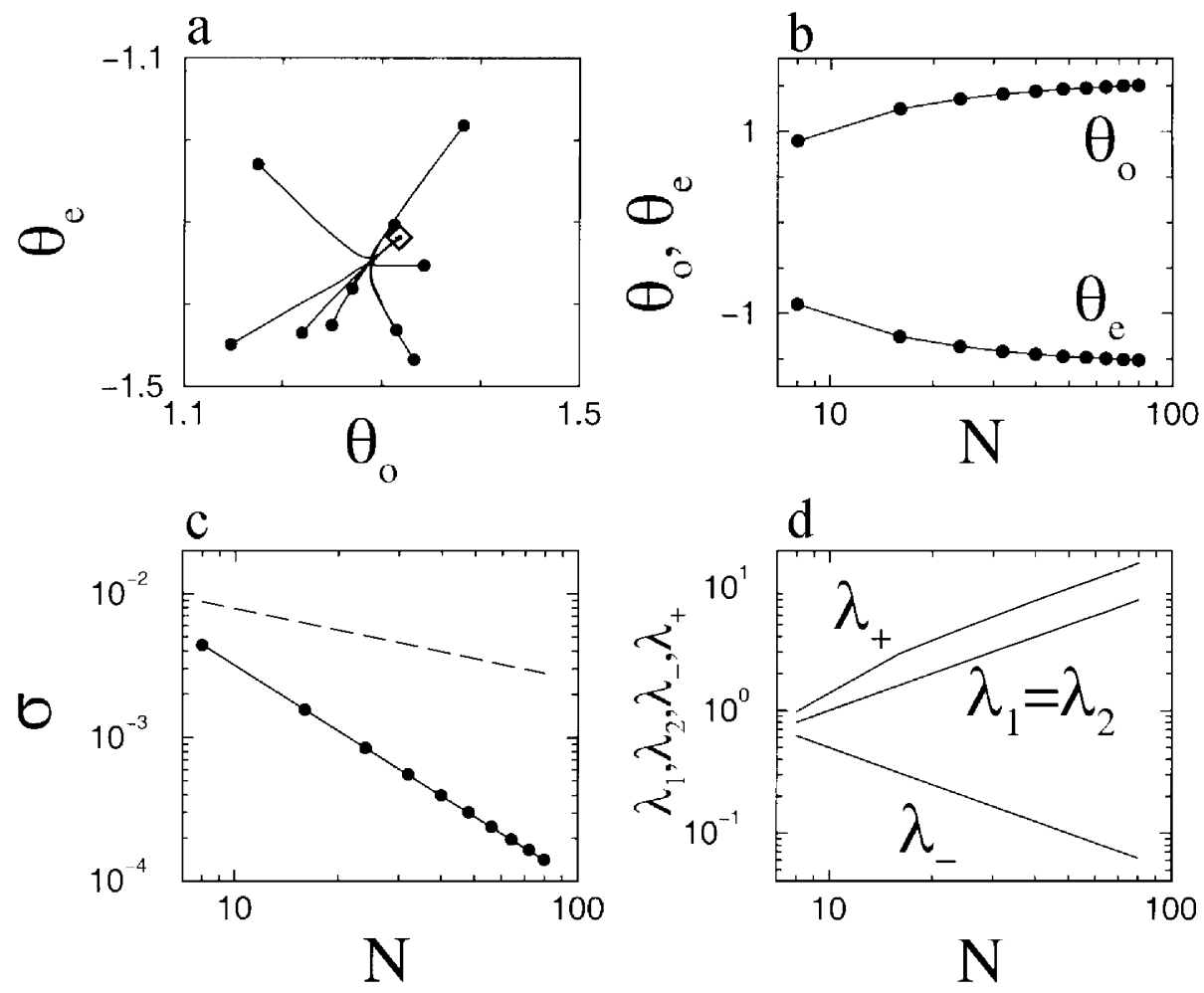

Fig. 2. Precision increased faster than $\sqrt{N}$ for extrinsic noise. (a) The network converged from random initial conditions to the fixed point, given by $\theta_{\mathrm{o}}=\beta_{1}=\pi / 2-\arcsin \left(1 / N K_{\mathrm{b}}\right)$ for all the odd phases, and $\theta_{\mathrm{e}}=\beta_{2}=-\pi / 2+\arcsin \left(1 / N K_{\mathrm{b}}\right)$ for the even phases. The $N=20$ neurons were divided into 10 even-odd pairs, the trajectory $\left(\theta_{\mathrm{o}}, \theta_{\mathrm{e}}\right)$ (solid line) was then plotted from the initial condition (circles) to the fixed point (open diamond). Here the rotating coordinate frame was used. (b) The fixed point phases $\theta_{\mathrm{o}}$ (top) and $\theta_{\mathrm{e}}$ (bottom) were plotted as a function of network size $N$ : the analytical result (solid line) and the simulation result (circles). (c) The analytical result (solid line, $Q_{\text {ex }} /\left(N^{3 / 2} K_{\mathrm{b}}\right)$ ) and numerical results (circles) for the jitter $\sigma$ as a function of $N$. For comparison the optimal result for intrinsic noise, $\sim 1 / N^{1 / 2}$, is plotted (dashed line). (d) Eigenvalues $\lambda$ of the stability matrix $\mathcal{H}_{i j}$ were plotted as a function of $N$. Analytical results, $\lambda_{1}=\lambda_{2}=\frac{1}{2} N K_{\mathrm{b}}, \lambda_{+}=N K_{\mathrm{b}}-1 / N K_{\mathrm{b}}$, and $\lambda_{-}=1 / N K_{\mathrm{b}}$. Eq. (13) were integrated using the Euler method with time step $\mathrm{d} t=1 \times 10^{-3}$, averages were over $10^{5}$ steps after discarding a transient of $2 \times 10^{4}$ steps. Parameter values were $Q_{\mathrm{ex}}=1 \times 10^{-2}, K_{\mathrm{b}}=0.2, K_{\mathrm{t}}=1, \Delta \omega=0$.

which is identical to Eq. (8) for two oscillators with $K_{\mathrm{b}} \rightarrow n K_{\mathrm{b}}$, hence we can use the solution given in Eqs. (9) and (10).

We transform to the rotating coordinate frame, $\theta_{i} \rightarrow \theta_{i}+\phi_{i}$. For notational simplicity, in the following and in Fig. $2, \theta_{i}, \theta_{\mathrm{o}}$ and $\theta_{\mathrm{e}}$, will denote the phase in the rotating coordinate frame. Hence, the phase dynamics of the $i$ th oscillator is

$$
\dot{\theta}_{i}=-\Delta \omega-K_{\mathrm{t}} \sin \left(\theta_{i}-\xi_{i, \mathrm{ex}}\right)+K_{\mathrm{b}} \sum_{j *} \sin \left(\theta_{j}-\theta_{i}-\left(\phi_{j}^{0}-\phi_{i}^{0}\right)\right) .
$$

The Hamiltonian (in the absence of noise) determined via $\dot{\theta}_{i}=-\partial \mathcal{H} / \partial \theta_{i}$ is

$$
\mathcal{H}=-\sum_{i}\left(-\Delta \omega \theta_{i}+K_{\mathrm{t}} \cos \left(\theta_{i}\right)\right)-K_{\mathrm{b}} \sum_{\{i, j\}} \cos \left(\theta_{j}-\theta_{i}+\Delta \phi\right),
$$

here $\sum_{\{i, j\}}$ is the sum over all pairs with $i$ odd and $j$ even. Solving the fixed point equations $\dot{\theta}_{i}=0$, yields $\theta_{i}=\beta_{1}$ for $i$ odd, and $\theta_{i}=\beta_{2}$ for $i$ even. Here $\beta_{1}$ and $\beta_{2}$ are given by Eqs. (9) and (10) with the substitution $K_{\mathrm{b}} \rightarrow n K_{\mathrm{b}}$. 
This fixed point is reached from random initial conditions (Fig. 2a). In the presence of sufficiently small noise, the arguments leading up to Eq. (3) can be repeated to yield,

$$
\delta \dot{\theta}_{i}=-\sum_{j} \mathcal{H}_{i j} \delta \theta_{j}+\cos \theta_{i} \xi_{i}
$$

and

$$
\sigma=\sqrt{\left\langle\left(\frac{1}{N} \sum_{i} \delta \dot{\theta}_{i}\right)^{2}\right\rangle}=\frac{Q_{\mathrm{ex}} K_{\mathrm{t}}}{N} \sqrt{\sum_{i} \cos ^{2} \theta_{i}}=\frac{Q_{\mathrm{ex}} K_{\mathrm{t}}}{N} \sqrt{\frac{N}{2}\left(\cos ^{2} \beta_{1}+\cos ^{2} \beta_{2}\right)} .
$$

In the limit $N K_{\mathrm{b}} \Delta \omega / K_{\mathrm{t}} \rightarrow 0$ and $K_{\mathrm{t}} / N K_{\mathrm{b}} \rightarrow 0$ the standard deviation of the jitter in the group rhythm becomes

$$
\sigma \approx \frac{K_{\mathrm{t}}^{2} Q_{\mathrm{ex}}}{N^{3 / 2} K_{\mathrm{b}}} \sim \frac{1}{N^{3 / 2}}
$$

This result was reproduced using numerical simulations (Fig. 2c). Therefore, with extrinsic noise the precision may improve as $N^{3 / 2}$, which is more rapid than the factor of $\sqrt{N}$ obtained with intrinsic noise. It is important to note that this $N^{3 / 2}$ improvement is only valid in a certain parameter regimes and does not hold in the asymptotic limit (except for $\Delta \omega=0$ ). As $N$ increases, the statement $N K_{\mathrm{b}} \Delta \omega / K_{\mathrm{t}} \rightarrow 0$ will eventually cease to be valid and thus the approximation leading to Eq. (20) will no longer hold. However, Eq. (20) may be valid for an arbitrarily large finite value of $N$ if the magnitude of $K_{\mathrm{b}}, K_{\mathrm{t}}$, and $\Delta \omega$ are chosen appropriately.

The solution is stable when all the eigenvalues of $\mathcal{H}_{i j} \equiv\left(\partial^{2} \mathcal{H} / \partial \theta_{i} \partial \theta_{j}\right)$ evaluated in the fixed point are positive. Substituting $\theta_{i}=\beta_{1}$ for $i$ is odd and $\theta_{i}=\beta_{2}$ otherwise, in the second derivative of the Hamiltonian Eq. (17) gives

$$
\mathcal{H}_{i j}=\left(\begin{array}{cccccccccc}
\beta_{0} & 0 & 0 & \ldots & 0 & \alpha & \ldots & \ldots & \ldots & \alpha \\
0 & \beta_{0} & 0 & \ldots & 0 & \alpha & \ldots & \ldots & \ldots & \alpha \\
& & & & & & & & & \\
0 & 0 & 0 & \ldots & \beta_{0} & \alpha & \ldots & \ldots & \ldots & \alpha \\
\alpha & \ldots & \ldots & \ldots & \alpha & \beta_{\mathrm{e}} & 0 & 0 & \ldots & 0 \\
\alpha & \ldots & \ldots & \ldots & \alpha & 0 & \beta_{\mathrm{e}} & 0 & \ldots & 0 \\
& & & & & & & & & \\
\alpha & \ldots & \ldots & \ldots & \alpha & 0 & 0 & 0 & \ldots & \beta_{\mathrm{e}}
\end{array}\right),
$$

where we used the following definitions,

$$
\alpha=-K_{\mathrm{b}} \cos \left(\beta_{2}-\beta_{1}+\Delta \phi\right), \quad \beta_{\mathrm{e}}=K_{\mathrm{t}} \cos \beta_{2}-n \alpha, \quad \beta_{\mathrm{o}}=K_{\mathrm{t}} \cos \beta_{1}-n \alpha .
$$

The determinant of $\mathcal{H}_{i j}-\lambda \delta_{i j}$ is for $N=2 n=6$ (the right-hand side is valid for any positive $n$ value),

$$
\operatorname{det}\left(\mathcal{H}_{i j}-\lambda \delta_{i j}\right)=\left|\begin{array}{cccccc}
\gamma_{0} & 0 & 0 & 0 & 0 & \alpha \\
-\gamma_{0} & \gamma_{0} & 0 & 0 & 0 & 0 \\
-2 \gamma_{0} & -\gamma_{0} & \gamma_{0} & 0 & 0 & 0 \\
0 & 0 & \alpha & \gamma_{\mathrm{e}} & 0 & 0 \\
0 & 0 & 0 & -\gamma_{\mathrm{e}} & \gamma_{\mathrm{e}} & 0 \\
0 & 0 & 0 & -2 \gamma_{\mathrm{e}} & -\gamma_{\mathrm{e}} & \gamma_{\mathrm{e}}
\end{array}\right|=\gamma_{0}^{n-1} \gamma_{\mathrm{e}}^{n-1}\left(\gamma_{\mathrm{e}} \gamma_{\mathrm{o}}-n^{2} \alpha^{2}\right)
$$

with $\gamma_{\mathrm{e}}=\beta_{\mathrm{e}}-\lambda, \gamma_{\mathrm{o}}=\beta_{\mathrm{o}}-\lambda$. The eigenvalues are obtained by setting the determinant to zero, $\lambda_{1}=\beta_{\mathrm{e}}(n-1$ eigenvectors), $\lambda_{2}=\beta_{\mathrm{o}}$ ( $n-1$ eigenvectors),

$$
\lambda_{ \pm}=\frac{1}{2}\left(\beta_{\mathrm{e}}+\beta_{\mathrm{o}}\right) \pm \frac{1}{2} \sqrt{\left(\beta_{\mathrm{e}}+\beta_{\mathrm{o}}\right)^{2}-4\left(\beta_{\mathrm{e}} \beta_{\mathrm{o}}-n^{2} \alpha^{2}\right)} .
$$


For small enough $K_{\mathrm{b}}$ and $N$ all eigenvalues are positive and the solution is stable. For $K_{\mathrm{t}}=1$ and $\Delta \omega=0$ the results simplify to, $\alpha=-K_{\mathrm{b}}\left(1-2 /\left(N K_{\mathrm{b}}\right)^{2}\right), \beta_{\mathrm{o}}=\beta_{\mathrm{e}}=\frac{1}{2} N K_{\mathrm{b}}, \lambda_{1}=\lambda_{2}=\frac{1}{2} N K_{\mathrm{b}}, \lambda_{+}=N K_{\mathrm{b}}-1 / N K_{\mathrm{b}}$, and $\lambda_{-}=1 / N K_{\mathrm{b}}$. These results are shown in Fig. $2 \mathrm{~d}$.

\section{Integrate-and-fire neurons}

We now demonstrate that the distinction between intrinsic and extrinsic noise is also valid for pulse-coupled integrate-and-fire (IAF) neurons, in addition to the simple phase models discussed previously. It has been shown that a $\sqrt{N}$ improvement in precision is observed for networks of IAF neurons with intrinsic additive current noise [20]. However, we will show that IAF neurons with extrinsic noise can have even greater precision than that obtained by simply averaging uncoupled IAF neurons.

We consider a model network of IAF neurons with the architecture pictured in Fig. 1. A neuron in the bottom layer has a membrane potential $V_{i}$ that obeys the equation,

$$
\dot{V}_{i}=I-V_{i}+E_{\mathrm{t}, i}(t)+\sum_{j}^{N} E_{\mathrm{b}, i, j}(t),
$$

where $I$ is a constant bias current, $E_{\mathrm{t}, i}(t)$ the synaptic current from the top layer to the $i$ theuron in the bottom layer, and $E_{\mathrm{b}, i, j}(t)$ the synaptic input from the $j$ th neuron in the bottom layer to neuron $i$. At the threshold $V_{i}=1$ the neuron produces a spike and then $V_{i}$ is reset to $0 . E_{\mathrm{b}, i, j}\left(t^{\prime}\right)=g_{\mathrm{b}} \alpha^{2} t^{\prime} \mathrm{e}^{-\alpha t^{\prime}}$ is an alpha function. Where $t^{\prime}$ is the time since the $j$ th neuron last fired, and $g_{\mathrm{b}}$ and $\alpha$ determine the strength and duration of the input. $E_{\mathrm{t}, i}(t)$ is a train of inhibitory delta function pulses that deliver a total current of $-g_{\mathrm{t}}$ at times $t=n T+\xi_{\mathrm{i}}$. Here $T$ is the period of oscillation of the neurons in the top layer, $\xi_{\mathrm{i}}$ is a white noise term with standard deviation $Q$, and $n=1,2,3, \ldots$ Thus, as in the phase model used above, the noise is jitter in the arrival of inputs from the top layer to the bottom layer. For the simulations in this paper $I=1.3, \alpha=4, g_{\mathrm{t}}=0.7, T=1.9$, and $Q=0.1$, unless noted otherwise. With these parameters the neurons in the bottom layer are phase locked to the neurons in the top layer, if the neurons in the bottom layer are uncoupled, $g_{\mathrm{b}}=0$. The qualitative nature of the results is not specific to these parameter values and remains the same if $E_{\mathrm{t}, i}(t)$ is changed to a series of alpha functions or $E_{\mathrm{b}, i, j}(t)$ is changed to a delta function.

As with the phase model, the noise introduced here is extrinsic because the fluctuations in the interspike intervals of the neurons in the bottom layer depends on the relative phase between these neurons and the neurons from the top layer. In this section we first demonstrate the extrinsic nature of the noise by setting $g_{\mathrm{b}}=0$ to consider the behavior of one IAF neuron uncoupled from the other neurons in the bottom layer. The isolated neuron in the bottom layer still receives a series of inhibitory delta function pulses from the top layer. The resulting fluctuations in the interspike intervals of the IAF neuron in the bottom layer depends on the magnitude of the jitter in the inhibitory input and on the relative phase between the input and the IAF neuron.

In the absence of noise, $Q=0$, there exists a solution to Eq. (24) for a range of driving currents $I$ with the neuron spiking periodically at time $T_{n}=n T$ and inhibitory pulses arriving at $t_{n}=\left(n-\phi_{0}\right) T$, where $\mathrm{n}$ is an integer [33]. The IAF neuron will asymptotically approach this stable phase-locked solution from an arbitrary initial condition. This approach to equilibrium can be described by a map $F$, which we derive in terms of the relative phase between the inhibitory input and the neurons' spike time. Given the previous spiking time $T_{n-1}$, and the arrival of the current pulse $\phi_{n}$, it is possible to determine the next spike time $T_{n}$. Since the next inhibitory input will arrive at time $T$ after the previous pulse, one can then determine its relative phase as $\phi_{n+1}=F\left(\phi_{n}\right)$. The resulting map is [34]

$$
F(\phi)=\phi-\frac{1}{T} \ln \left[1+\frac{g_{\mathrm{t}}}{I+g_{\mathrm{t}} / \alpha_{0}}\left(\frac{1}{\alpha}-\frac{1}{\alpha_{0}}\right)\right] .
$$



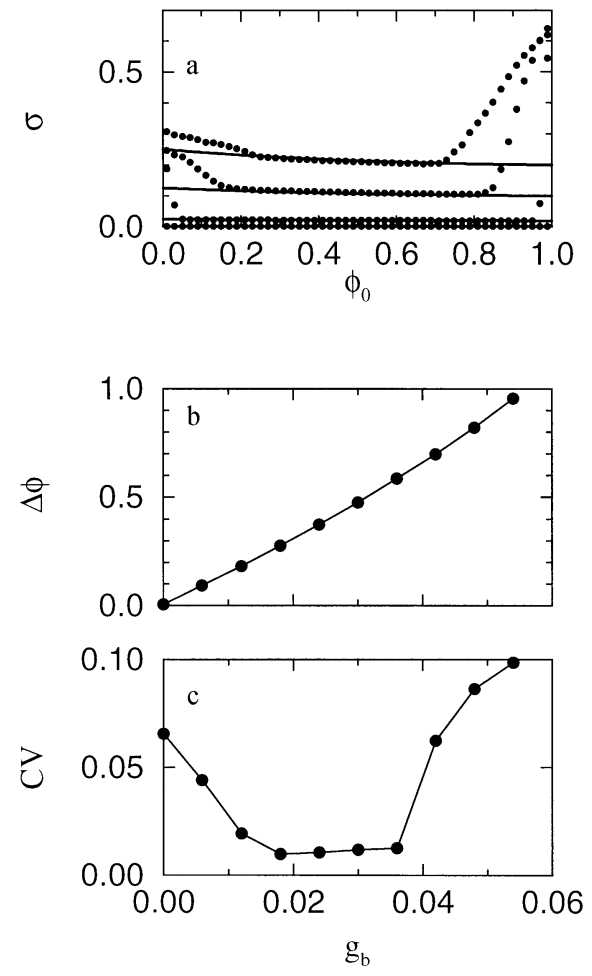

Fig. 3. (a) The output jitter of a single IAF neuron in the bottom layer as a function of $\phi_{0}$. The magnitude of the extrinsic noise varies from top to bottom as $Q=0.1,0.05,0.01$ and 0.001 . The small circles are obtained from numerical simulation of the map with noise, where as the solid lines are the solution of the linearized dynamics. For a network of $N=10$ IAF neurons described by Eq. (24) the relative phase $\Delta \phi$ between the bottom layer and the input from the top layer, and the $\mathrm{CV}$, are both functions of $g_{\mathrm{b}}$. (b) The mean relative phase between the input from the top layer and the bottom layer, as a function of $g_{\mathrm{b}}$ in the absence of noise. (c) $\mathrm{CV}$ as a function of $g_{\mathrm{b}}$ with $Q=0.01$. The increase of $\mathrm{CV}$ at high and low $g_{\mathrm{b}}$ (high and low relative phase) is due to skipping.

Here $\alpha=\mathrm{e}^{-\phi T}, \alpha_{0}=\mathrm{e}^{-\phi_{0} T}, I=I_{0}+\left(g_{\mathrm{t}} / \alpha_{0}\right) \delta /(1-\delta), I_{0}=1 /(1-\delta)$, and $\delta=\mathrm{e}^{-T}$. To obtain a given fixed point $\phi_{0}$ of Eq. (25), one has to inject a specific current value.

Now consider the effect of jitter in the arrival time of the input pulses by making $Q$ nonzero. One can then determine the resulting jitter in the spike time $T_{n}$ and the interspike intervals $\tau_{n}=T_{n+1}-T_{n}$. An estimate of $\sigma$, the resulting standard deviation in the interspike interval of the IAF neuron, can be obtained by linearizing the map,

$$
\sigma=\sqrt{\frac{\chi}{2-\chi}} Q
$$

Here $1-\chi$ is the derivative of the map at the fixed point phase, $\chi=1-\left.(\mathrm{d} F / \mathrm{d} \phi)\right|_{\phi_{0}}=g_{\mathrm{t}} /\left(I \alpha_{0}+g_{\mathrm{t}}\right)$. The value of $\sigma$ is a function of $\phi_{0}$, the equilibrium phase between the input and the IAF neuron, and thus the noise is indeed extrinsic. The analytical results from Eq. (26) are plotted for various values of $Q$ and compared to computer simulations in Fig. 3(a). For intermediate values of $\phi_{0}$ there is excellent agreement between the theory and the simulation. However, as $\phi_{0}$ approaches 0 or 1 the theory significantly underestimates the resulting jitter of the IAF neuron.

This failure of the theory can be understood by noting that the linearized map will only provide a good estimate of $\sigma$ if the IAF neuron spikes only once after each inhibitory input. If $Q$ is large enough, the next inhibitory pulse can arrive immediately after the previous one, before the IAF neuron can spike. This happens when $\phi_{0}$ is close to 

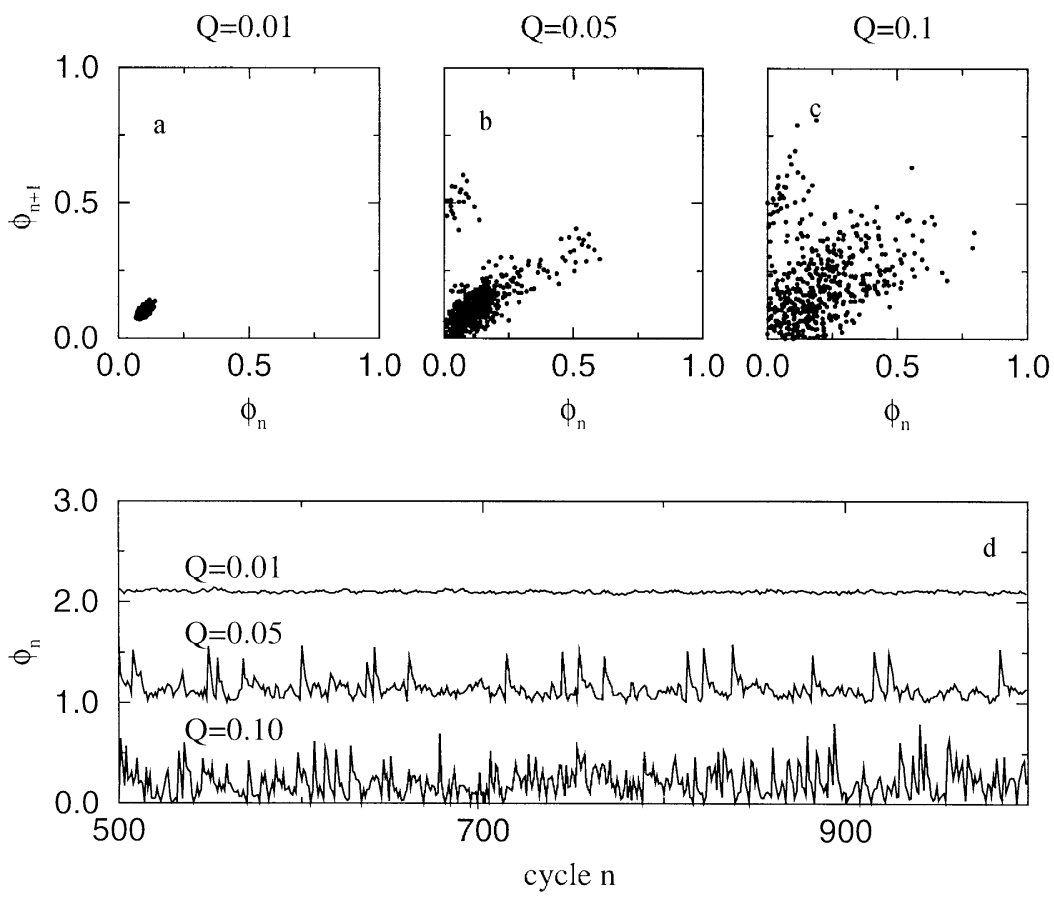

Fig. 4. We plot the return map $\phi_{n+1}$ versus $\phi_{n}$ obtained from numerical simulation for: (a) $Q=0.01$, (b) $Q=0.05$ and (c) $Q=0.10$. In (d) we plot the actual time trace, the $\phi_{n}$ as a function of the cycle number $n$ for the same values of $Q$ as labeled in the figure. For clarity the phases for $Q=0.01$ and 0.05 are offset by 2 and 1, respectively. We take $\phi_{0}=0.1$, the other parameters are as described in the text.

one. Similarly, if $\phi_{0}$ is close to zero the IAF neuron can spike twice before the next inhibitory input arrives. In both cases the phase $\phi_{n+1}$ of the new pulse is far from the fixed point value of $\phi_{0}$. Over the next few iterations of the map it will again approach the fixed point. However, these spike skipping and spike missing events do significantly increase $\sigma$. We illustrate this phenomena in Fig. 4 where we plot the return map, $\phi_{n+1}$ versus $\phi_{n}$, obtained from numerical simulations for various values of $Q$.

If $g_{\mathrm{b}} \geq 0$ the neurons in the bottom layer are coupled via excitatory pulses, which provide an extra, phasic, driving current. In this case, the relative phase between the neurons in the bottom layer and the input from the top layer depends on the strength of the coupling and thus the amount of jitter in the output depends on the strength of coupling. Fig. 3(b) and (c) show how the relative phase and the amount of jitter changes as $g_{\mathrm{b}}$ is varied for a network with $N=10$ neurons in the bottom layer. This is analogous to the behavior of a single IAF neuron shown in Fig. 3(a). From this graph it can be seen that, for a given number of neurons, a value of $g_{b}$ can be selected to minimize the amount of jitter.

In a similar fashion, for a given value of $g_{\mathrm{b}}$ there is a particular number of neurons that minimizes the amount of jitter. For example, if for a particular number of neurons in the network, the neurons are phase locked to the input with $\phi_{0}$ close to zero, adding more neurons to the network will increase $\phi_{0}$ and thus decrease the $\sigma$ of each IAF neuron. Therefore, the $\mathrm{CV}$ of the group rhythm can be decreased to better than $1 / \sqrt{N}$ times the $\mathrm{CV}$ of an individual oscillator. This is depicted in Fig. 5, where the CV is plotted versus the number of neurons in the bottom layer, with $g_{\mathrm{b}}=3 \times 10^{-3}$. This figure clearly demonstrates an improvement in precision in addition to $\sqrt{N}$. When more neurons are added the improvement in jitter levels off and for larger network sizes the precision of the group rhythm may be worse than $\sqrt{N}$ times the precision of an uncoupled neuron. However, for any given number of neurons in the network, $N_{\max }$, it is always possible to choose a coupling strength, $g_{\mathrm{b}}$, such that as $N$ increases from 1 to $N_{\max }$, the $\mathrm{CV}$ of the network improves by more than $1 / \sqrt{N}$ for all $N$ less than $N_{\max }$. 


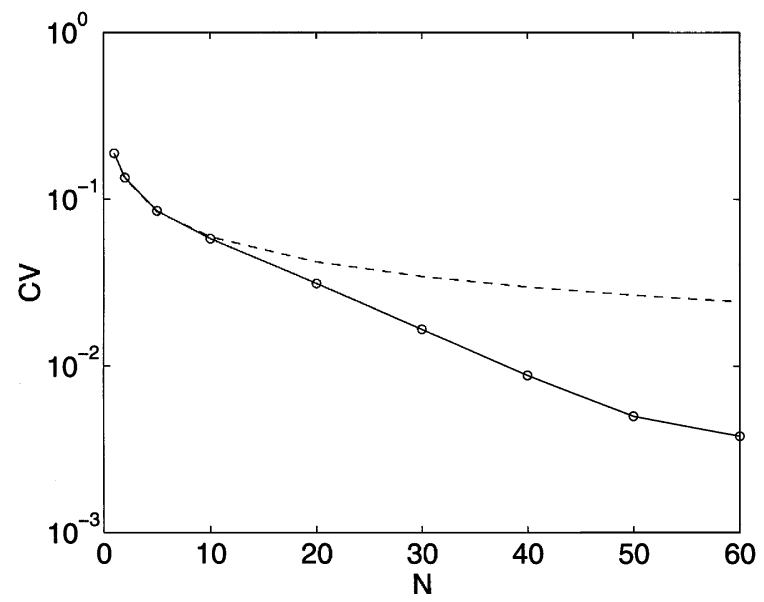

Fig. 5. The CV of the group rhythm of a network of IAF neurons with dynamics described by Eq. (24) as a function of the number of neurons in the network, $N, g_{\mathrm{b}}=3 \times 10^{-3}$ and other parameter values are as described in the text. The circles are values obtained by solving Eq. (24) numerically with Euler's method with a time step of $\mathrm{d} t=1 \times 10^{-3}$. The dashed line is the CV of an uncoupled neuron times $1 / \sqrt{N}$. In this range, the precision of the group rhythm is better than $\sqrt{N}$ times the precision of an uncoupled neuron. As the number of neurons is increased further, the $\mathrm{CV}$ of the neurons stops decreasing with $N$. When $N$ becomes sufficiently large, the precision becomes worse than $\sqrt{N}$ times the precision of an uncoupled neuron.

\section{Conclusion}

In summary, we have demonstrated in two model systems that it is possible to enhance the precision of a set of oscillators beyond $\sqrt{N}$ through coupling but only when the sources of noise are extrinsic rather than intrinsic to the oscillators. The group rhythm of intrinsically noisy oscillators is more precise than an individual uncoupled oscillator because the fluctuations in the individual oscillators cancel by the law of large numbers. For extrinsically noisy oscillators, coupling may make the extrinsic noise term have less of an effect, resulting in a group rhythm with a standard deviation that is even better than that obtained by averaging. It should be borne in mind, however, that all the results obtained here were for finite $N$; it remains to explore whether similar improvements are present asymptotically for large $N$ [35]. This could occur through reducing the multiplicative constant for $1 / \sqrt{N}$ or by finding circumstances when the falloff is even steeper than $1 / \sqrt{N}$.

Finally, the results obtained here may have biological significance for systems of neurons that need to be precisely synchronized [16,17]. Intrinsic noise cannot be reduced beyond $1 / \sqrt{N}$, so the only way to achieve high precision is to use mechanisms that have low intrinsic noise, which appears to be the case for the pacemaker nucleus of weakly electric fish [18,19]. Synaptic noise is present in many cortical neurons [36] and it may be possible to improve the precision of their synchronization, such as that found during sleep rhythms [37], since synaptic variability is a form of extrinsic noise.

\section{Acknowledgements}

We would like to thank Maxim Bazhenov, Michael Eisele, and Alexei Koulakov for their advice. We thank Steven Strogatz for instructive conversations about collective enhancement of precision. We appreciate and enjoyed our discussion of the manuscript with Alan Needleman. We thank Bruce Knight for encouragement and thoughtful comments on an earlier version of the manuscript. 


\section{References}

[1] A.T. Winfree, The Geometry of Biological Time, Springer, New York, 1980.

[2] C. Liu, D. Weaver, S.H. Strogatz, S.M. Reppert, Cell 91 (1997) 855-860.

[3] C.M. Gray, J. Comput. Neurosci. 1 (1994) 11-38.

[4] D. Hansel, G. Mato, C. Meunier, Europhys. Lett. 23 (1993) 367-372.

[5] D. Hansel, G. Mato, C. Meunier, Neural Comput. 7 (1995) 307-337.

[6] K. Wiesenfeld, P. Colet, S.H. Strogatz, Phys. Rev. Lett. 76 (1996) 404-407.

[7] A.T. Winfree, J. Theoret. Biol. 16 (1967) 15-42.

[8] T.L. Williams, K.A. Sigvardt, N. Kopell, G.B. Ermentrout, M.P. Remler, J. Neurophys. 64 (1990) 862-871.

[9] G.B. Ermentrout, J. Flores, A. Gelperin, J. Neurophys. 79 (1998) 2677-2689.

[10] S.H. Strogatz, in: S. Levin (Ed.), Frontiers in Mathematical Biology, Lecture Notes in Biomathematics, Vol. 100, Springer, Berlin, 1994.

[11] Y. Kuramoto, Chemical Oscillations, Waves, and Turbulence, Springer, Berlin, 1984.

[12] R.L. Stratonovich, Topics in the Theory of Random Noise, Vol. II, Gordon and Breach, New York, 1967 (Chapter 9).

[13] J.D. Hunter, J.G. Milton, P.J. Thomas, J.D. Cowan, J. Neurophysiol. 80 (1998) 1427-1438.

[14] P.H.E. Tiesinga, J.V. José, Network: Comput. Neural Syst. 11 (2000) 1-23.

[15] J. Enright, Science 209 (1980) 1542-1545.

[16] J. Enright, The Timing of Sleep and Wakefulness, Springer, Berlin, 1980.

[17] K.T. Moortgat, C.H. Keller, T.H. Bullock, T.J. Sejnowski, Proc. Natl. Acad. Sci. USA 95 (1998) 4684-4689.

[18] K.T. Moortgat, T.H. Bullock, T.J. Sejnowski, J. Neurophysiol. 83 (2000) 971-983.

[19] K.T. Moortgat, T.H. Bullock, T.J. Sejnowski, J. Neurophysiol. 83 (2000) 984-997.

[20] W.J. Rappel, A. Karma, Phys. Rev. Lett. 77 (1996) 3256-3259.

[21] T. Tateno, Y. Jimbo, Biol. Cybernetics 80 (1999) 45-55.

[22] J.G. Maneatis, M.A. Horowitz, ISSC 1993 Dig. Tech. Papers, 1993, pp. 118-119.

[23] J.G. Maneatis, M.A. Horowitz, IEEE J. Solid-State Circ. 28 (1993) 1273-1282.

[24] G.B. Ermentrout, N. Kopell, SIAM J. Math. Anal. 15 (1984) 215-237.

[25] G.B. Ermentrout, D. Kleinfeld, Neuron 29 (2001) 33-44.

[26] J. Rinzel, G.B. Ermentrout, in: C. Koch, I. Segev (Eds.), Methods in Neuronal Modeling: From Synapses to Networks, MIT press, Cambridge, MA, 1998, pp. 251-291.

[27] P.C. Bressloff, S. Coombes, Neural Comput. 12 (2000) 91-129.

[28] C. van Vreeswijk, L.F. Abbott, G.B. Ermentrout, J. Comput. Neurosci. 1 (1994) 313-321.

[29] S.H. Strogatz, Personal communication.

[30] T. Shinbrot, K. Scarbrough, J. Theoret. Biol. 196 (1999) 455-471.

[31] J.R. Clay, R.L. DeHann, Biophys. J. 28 (1979) 377-389.

[32] Z.F. Mainen, T.J. Sejnowski, Science 268 (1995) 1503-1506.

[33] S. Coombes, P.C. Bressloff, Phys. Rev. E 60 (1999) 2086-2096.

[34] C. Bernasconi, K. Schindler, R. Stoop, R. Douglas, Neural Comput. 11 (1999) 67-74.

[35] P.H.E. Tiesinga, T.J. Sejnowski, Network 12 (2001) 215.

[36] V.N. Murthy, T.J. Sejnowski, C.F. Stevens, Neuron 18 (1997) 599-612.

[37] M. Steriade, D.A. McCormick, T.J. Sejnowski, Science 262 (1993) 679-685. 